\title{
Indicadores
}

\section{La productividad ISI de las universidades españolas (2000-2004)}

\author{
Por Grupo Scimago
}

\begin{abstract}
Resumen: Cuantificación de la investigación universitaria desde que comenzó la convergencia hacia el Espacio Europeo de Educación Superior. El sistema universitario representa el 66,43\% sobre el total de producción científica española con visibilidad internacional en el quinquenio 2000-04, medida a través del Web of Science. Setenta universidades españolas tienen una producción visible internacionalmente. De ellas, 36 con más de 1.000 documentos. Las universidades ubicadas en tres comunidades autónomas: Cataluña, Madrid y Andalucía, acumulan el 58\% del total académico.
\end{abstract}

Palabras clave: Produción científica, Universidades españolas, Web of Sciece, Índice de productividad, Ranking, España.

\section{Title: ISI productivity of the Spanish Universities (2000-2004)}

Abstract: Quantification of the university research since the convergence towards the European Higher Education Area (EHEA) began. The university system represents the 66,43\% on the total Spanish scientific production with international visibility in the five-year period 2000-04, measured through the Web of Science. Seventy Spanish universities have an internationally visible production. Of them, 36 have more than 1,000 documents. The universities located in three Spanish regions: Catalonia, Madrid and Andalusia, accumulate the $58 \%$ of the academic total.

Keywords: Scientific productivity, Spanish universities, Web of Science, Productivity index, Ranking, Spain

Grupo Scimago. La productividad ISI de las universidades españolas (2000-2004) En: El profesional de la información, 2007, julio-agosto, v. 16. n.4, pp. 354-358.

DOI: 10.3145/epi.2007.jul.11

LAS UNIVERSIDADES, como centros de enseñanza superior que confieren grados académicos, además de ejercer una indiscutible labor docente, destacan por su papel investigador. Esta actividad fue un aspecto tratado en la reunión de ministros europeos celebrada en Bolonia en el año 1999, que abrió paso al proceso de convergencia que llevará a las instituciones de educación superior a formar un único espacio europeo de enseñanza. Se va a promover tanto la calidad docente como la de la investigación que se realiza dentro de dicho espacio y se establecerán mecanismos para su medida ${ }^{1}$.

Este trabajo cuantifica la investigación universitaria a partir de la fecha en la que se comenzó la convergencia hacia el Espacio Europeo de Educación Superior. El proceso continúa actualmente abierto y en él se encuentra inmerso el principal sector productivo en los distintos campos temáticos: el sector universitario $^{2}$. El sistema universitario representa el $66,43 \%$ sobre el total de producción científica española con visibilidad internacional en el período.

La producción de las universidades españolas se ha medido a través de los datos recogidos en el Web of Science ${ }^{3}$, en las bases de datos Science Citation Index-Expandex (SCI-E), Social Science Citation Index (SSCI) y Arts \& Humanities Citation Index (A\&HCI), desde el año 2000 hasta el 2004, ambos incluidos. Esta fuente de datos ya se ha probado para medir un único dominio institucional universitario ${ }^{4}$, ${ }^{5} \mathrm{y}$ como recurso empleado en métodos avanzados de la evaluación de las universidades ${ }^{6}$, por lo que se estima válida para la evaluación de los resultados de investigación del sistema universitario español.

Setenta universidades españolas tienen una producción visible en una fuente de datos internacional y cada uno de los centros estudiados alcanza diferentes valores. De ellos, 36 instituciones universitarias tienen una producción que supera los 1.000 documentos en el quinquenio. Las universidades ubicadas en tres comunidades autónomas, Cataluña, Madrid y Andalucía, acumulan el 58\% de la producción universitaria.

\section{Índice de productividad (Iprod)}

Si se estableciera un orden, o ranking, teniendo en cuenta tan solo la variable de la producción científica, se posicionarían en los 
1) Blogs y otros productos informativos en internet.

2) Organización de cursos.

a) Propios abiertos al público en general.

b) Para empresas, como formación interna o mediante la externalización de su propuesta formativa (co-organización).

c) Para colectivos que necesiten formación espećíica.

3) Organización de jornadas y otros eventos profesionales.

4) Selección de personal.

\section{www.informa.cat}


puestos más altos aquellos centros que tienen una alta producción. Como ya han demostrado estudios previos $^{7}$, es interesante combinarlo con otros indicadores. El objetivo de este trabajo es obtener un indicador de rendimiento de las universidades basado en la producción de documentos con visibilidad internacional existentes en el Web of Science. Por esa razón la tabla 1 recoge por estricto orden alfabético todas las instituciones universitarias con producción visible en el ámbito internacional, con el total de su producción en el quinquenio (Ndoc), el promedio del profesorado de cada universidad además de su Índice de productividad (Iprod) normalizado.

Los datos de profesorado han sido extraídos de las estadísticas oficiales publicadas por años académicos por el Instituto Nacional de Estadística (INE) ${ }^{8}$ y para el cálculo del Iprod recogido en la tabla 1 , se tuvo en cuenta el dato de profesorado con dedicación a tiempo completo, tanto de universidades públicas como de universidades privadas, desde el curso 1999-2000 hasta el 2003-2004. Según las indicaciones del INE, se debe aclarar que el profesorado a dedicación a tiempo completo de la Universidad de Navarra incluye el personal docente del Hospital Universitario de Navarra.

El Iprod se obtiene dividiendo la producción científica con visibilidad internacional de cada centro, entre el número de profesores con dedicación a tiempo completo de la institución. El cálculo que se recoge en la tabla 1, es el Iprod total de una universidad en el periodo, es decir, la suma de los Iprod calculados para cada centro desde el año 2000 hasta el 2004. La Universidad Católica San Vicente Mártir y la Universidad Internacional Menéndez. Pelayo no tienen cálculo del Iprod debido a que el INE para estos centros no facilita datos de su profesorado, en ninguno de los años estudiados. Hay seis universidades que, en alguno de estos cinco años, o no tuvieron producción internacional, o el INE no facilita datos de su profesorado. En esos casos se realiza una estimación utilizando el Iprod alcanzado en los años en los que sí se dispone de los datos necesarios para su cálculo (indicado con * en la tabla 1).

\section{Iprod normalizado}

Con vistas a que este indicador pueda ser comparado con otros indicadores en escalas similares, se ha procedido a su normalización. Un procedimiento posible que permite conservar cualquier sesgo de la distribución original, consiste en dividir el valor del indicador de una universidad por el valor máximo del indicador en toda la serie de universidades. Así la universidad con la mejor puntuación obtiene el valor de 1, mientras las otras universidades obtendrán valores entre 0 y 1, dependiendo del valor de su indicador. En este caso, ese proceso supone dividir los Iprod de cada una de las universidades por el valor máximo obtenido por un centro universitario determinado.

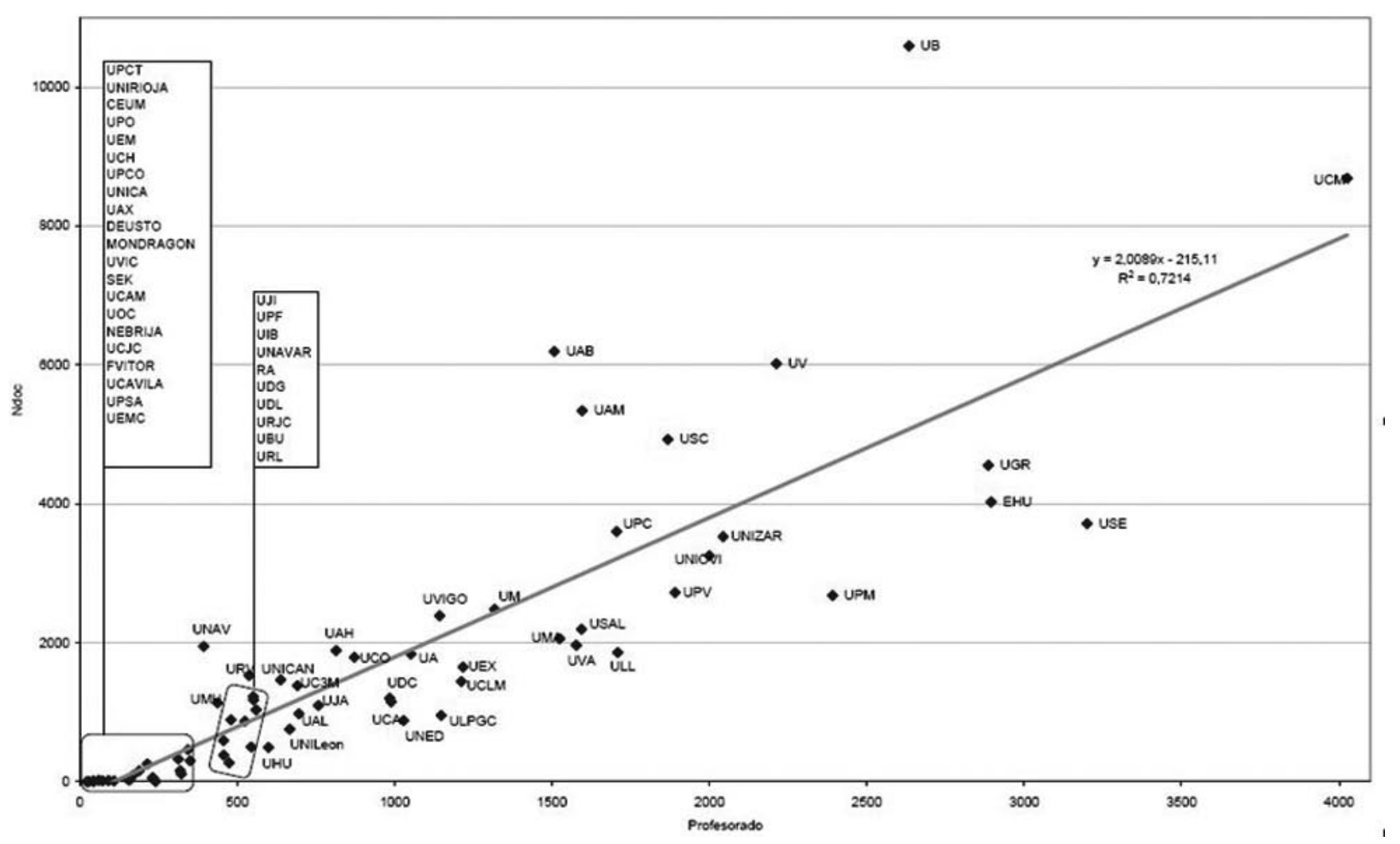

Gráfico 1. Dispersión y regresión lineal de la producción y el profesorado con dedicación a tiempo completo (2000-2004) 


\begin{tabular}{|c|c|c|c|}
\hline Universidad & Ndoc & Profs. & Iprod \\
\hline UNED & 882 & 1.029 & 0,20 \\
\hline Univ Alcalá de Henares UAH & 1.888 & 814 & 0,53 \\
\hline Univ Alfonso X el Sabio UAX & 52 & 232 & 0,08 \\
\hline Univ Antonio de Nebrija NEBRIJA & 10 & 108 & 0,03 \\
\hline Univ Autónoma de Barcelona UAB & 6.195 & 1.507 & 0,94 \\
\hline Univ Autónoma de Madrid UAM & 5.341 & 1.596 & 0,77 \\
\hline Univ Camilo José Cela UCJC & 9 & 43 & 0,10 \\
\hline Univ Cardenal Herrera CEU UCH & 159 & 189 & 0,24 \\
\hline Univ Carlos III UC3M & 1.384 & 692 & 0,45 \\
\hline Univ Católica Ávila UCAVILA & 4 & 28 & 0,08 \\
\hline Univ Católica San Antonio UCAM & 16 & 90 & 0,04 \\
\hline Univ Católica San Vicente Mártir & 2 & - & - \\
\hline Univ Complutense UCM & 8.690 & 4.026 & 0,49 \\
\hline Univ da Coruna UDC & 1.203 & 985 & 0,28 \\
\hline Univ d'Alacant UA & 1.837 & 1.051 & 0,40 \\
\hline Univ de Almería UAL & 978 & 696 & 0,33 \\
\hline Univ de Barcelona UB & 10.596 & 2.634 & 0,92 \\
\hline Univ de Burgos UBU & 382 & 457 & 0,19 \\
\hline Univ de Cádiz UCA & 1.148 & 989 & 0,26 \\
\hline Univ de Cantabria UNICAN & 1.466 & 638 & 0,53 \\
\hline Univ de Castilla La Mancha UCLM & 1.444 & 1.212 & 0,27 \\
\hline Univ de Córdoba UCO & 1.792 & 872 & 0,47 \\
\hline Univ de Deusto DEUSTO & 52 & 231 & 0,07 \\
\hline Univ de Extremadura UEX & 1.651 & 1.217 & 0,32 \\
\hline Univ de Girona UDG & 872 & 524 & 0,38 \\
\hline Univ de Granada UGR & 4.557 & 2.886 & 0,36 \\
\hline Univ de Huelva UHU & 493 & 599 & 0,19 \\
\hline Univ de Jaén UJA & 1.099 & 758 & 0,33 \\
\hline Univ de La Rioja UNIRIOJA & 326 & 313 & 0,24 \\
\hline $\begin{array}{l}\text { Univ de las Palmas Gran Canaria } \\
\text { ULPGC }\end{array}$ & 954 & 1.148 & 0,19 \\
\hline Univ de León UNILeon & 758 & 667 & 0,26 \\
\hline Univ de les Illes Balears UIB & 1.034 & 560 & 0,42 \\
\hline Univ de Lleida UDL & 594 & 457 & 0,30 \\
\hline Univ de Málaga UMA & 2.060 & 1.524 & 0,31 \\
\hline Univ de Mondragón MONDRAGON & 31 & 153 & 0,04 \\
\hline
\end{tabular}

\begin{tabular}{|c|c|c|c|}
\hline Universidad & Ndoc & Profs. & Iprod \\
\hline Univ de Murcia UM & 2.483 & 1.317 & 0,43 \\
\hline Univ de Navarra UNAV & 1.948 & 394 & 1,00 \\
\hline Univ de Oviedo UNIOVI & 3.253 & 2.000 & 0,39 \\
\hline Univ de País Vasco EHU & 4.028 & 2.895 & 0,32 \\
\hline Univ de Salamanca USAL & 2.197 & 1.593 & 0,32 \\
\hline $\begin{array}{l}\text { Univ de Santiago de Compostela } \\
\text { USC }\end{array}$ & 4.926 & 1.868 & 0,61 \\
\hline Univ de Sevilla USE & 3.715 & 3.199 & 0,27 \\
\hline Univ de Valencia UV & 6.020 & 2.212 & 0,62 \\
\hline Univ de Valladolid UVA & 1.964 & 1.577 & 0,28 \\
\hline Univ de Vic UVIC & 26 & 158 & 0,04 \\
\hline Univ de Vigo UVIGO & 2.390 & 1.142 & 0,49 \\
\hline Univ de Zaragoza UNIZAR & 3.528 & 2.043 & 0,39 \\
\hline Univ Europea Madrid CEES UEM & 160 & 319 & 0,11 \\
\hline $\begin{array}{l}\text { Univ Europea Miguel de Cervantes } \\
\text { UEMC }\end{array}$ & 1 & 22 & 0,06 \\
\hline Univ Francisco de Vitoria FVITOR & 5 & 44 & 0,12 \\
\hline $\begin{array}{l}\text { Univ Internacional Catalunya } \\
\text { UNICA }\end{array}$ & 89 & 169 & 0,20 \\
\hline $\begin{array}{l}\text { Univ Internacional Menéndez } \\
\text { Pelayo }\end{array}$ & 1 & - & - \\
\hline Univ Jaume I UJI & 1.225 & 551 & 0,50 \\
\hline Univ La Laguna ULL & 1.861 & 1.709 & 0,26 \\
\hline Univ Miguel Hernández UMH & 1.134 & 437 & 0,59 \\
\hline Univ Oberta Catalunya UOC & 12 & 72 & 0,04 \\
\hline Univ Pablo de Olavide UPO & 254 & 214 & 0,25 \\
\hline $\begin{array}{l}\text { Univ Politécnica de Cartagena } \\
\text { UPCT }\end{array}$ & 463 & 343 & 0,30 \\
\hline Univ Politécnica de Catalunya UPC & 3.601 & 1.705 & 0,49 \\
\hline Univ Politécnica de Madrid UPM & 2.680 & 2.391 & 0,26 \\
\hline Univ Politécnica de Valencia UPV & 2.725 & 1.891 & 0,33 \\
\hline Univ Pompeu Fabra UPF & 1.180 & 552 & 0,50 \\
\hline Univ Pontificia Comillas UPCO & 109 & 322 & 0,10 \\
\hline Univ Pontificia Salamanca UPSA & 2 & 240 & 0,01 \\
\hline $\begin{array}{l}\text { Univ Pública de Navarra } \\
\text { UNAVARRA }\end{array}$ & 890 & 480 & 0,43 \\
\hline Univ Ramon Llul URL & 270 & 474 & 0,15 \\
\hline Univ Rey Juan Carlos I URJC & 500 & 544 & 0,20 \\
\hline Univ Rovira i Virgili URV & 1.530 & 537 & 0,67 \\
\hline Univ San Pablo CEU CEUM & 301 & 350 & 0,19 \\
\hline Univ Sek SEK & 21 & 59 & 0,11 \\
\hline
\end{tabular}

Tabla 1. Producción, promedio del profesorado con dedicación completa e lprod de las universidades españolas (2000-2004) 


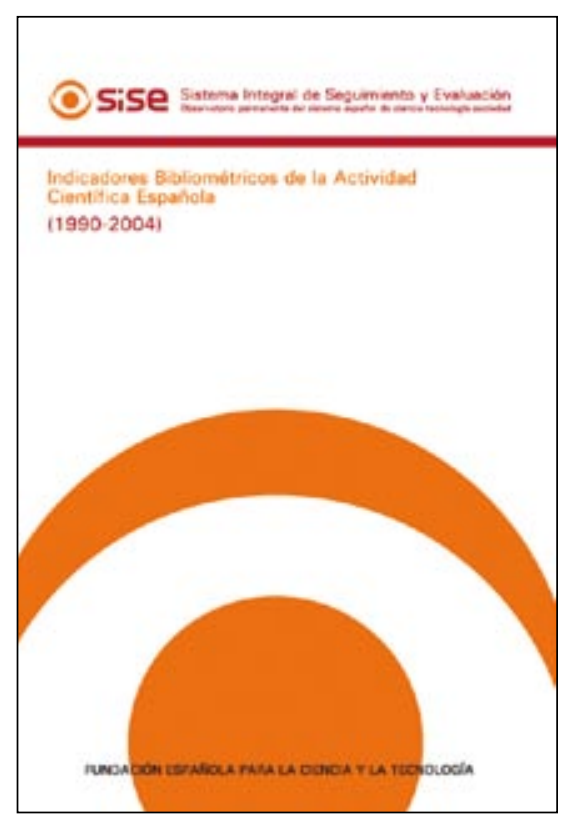

Grupo Scimago. Indicadores bibliométricos de la actividad científica española (19902004) [dirección Félix de Moya-Anegón, coordinación Zaida Chinchilla-Rodríguez, equipo de investigación: Elena CoreraÁlvarez, María-del-Rocío GómezCrisóstomo (Univ. de Extremadura), Antonio González-Molina, FranciscoJosé Muñoz-Fernández, y Benjamín Vargas-Quesada. Madrid: Fecyt. http://sise.fecyt.es/Estudios/Doc/ indicadores_bibliometricos.pdf

El total de la columna de producción universitaria internacional alcanza 115.451 documentos, siendo 64.750 la suma de la media del profesorado con dedicación a tiempo completo. El Iprod total nacional de las universidades en el periodo es de 1,56 .

Si se realiza un ranking según la producción de los centros, las cuatro universidades más productivas, todas con más de 6.000 documentos publicados entre el año $2000 \mathrm{y}$ el año 2004, aparece en primer lugar la Universidad de Barcelona, seguida de la Universidad Complutense, la Universidad Autónoma de Barcelona y la Universidad de Valencia. Si ese orden se establece en función del Iprod normalizado de las instituciones incluidas en este estudio, la Universidad de Navarra consigue el máximo valor del índice y a continuación se posicionan la Universidad Autónoma de Barcelona, la Universidad de Barcelona, la Universidad Autónoma de Madrid, la Universidad de Santiago de Compostela y la Universidad de Valencia.

Del mismo modo que el Iprod relaciona la productividad de los centros con su profesorado, el gráfico 1 muestra un diagrama de dispersión y la regresión lineal que representa el binomio de la producción absoluta en el quinquenio y el promedio del número de profesores con dedicación a tiempo completo en el período estudiado (20002004). En el eje $X$ se representa el número de profesores y en el eje $\mathrm{Y}$ la producción absoluta de cada universidad. Ambos ejes correlacionan con un $\mathrm{R}^{2}=0,72$. La línea continua representa la regresión lineal. Los cuadrados representan los centros. Para establecer la correspondencia entre las abreviaturas que se muestran en el gráfico y las universidades, se ha de consultar la tabla 1 . Así se puede resaltar que la Universidad de Barcelona es el centro con más producción en el período, aunque no es la universidad que mayor número de profesores tiene. Esa característica le corresponde a la Universidad Complutense de Madrid. El resto de universidades más productivas cuentan con un dotación de profesorado que oscila entre los 1.500 y 2.200 profesores con dedicación a tiempo completo.

Después de haber realizado una aproximación cuantitativa a la producción universitaria de las universidades españolas en cinco años y su relación con el factor directa- mente implicado, el profesorado, en próximos artículos sería interesante cualificar esta producción y se hará sustituyendo la variable de producción bruta por la de producción ponderada en función de la calidad de las revistas en que han sido publicados los diferentes trabajos universitarios.

\section{Notas}

1. Braun, T. "Bibliometric indicators for the evaluation of universities - intelligence from de quantification of the scientific literature". En: Scientometrics, 1999, v. 45, n. 3, pp. 425-432.

2. Grupo Scimago. "Ranking de instituciones más productivas del sector sanitario español". En: El profesional de la información, 2005, v. 14 , n. 5 , pp. $347-348$.

3. Web of Knowledge (WoK). Disponible: http://www.accesowok.fecyt.es [Consulta: 01-122006].

4. Moya-Anegón, F; Chinchilla-Rodríguez, Z.; Corera-Álvarez, E.; Vargas-Quesada, B.; Muñoz-Fernández, F. J.; Herrero-Solana, V. "Análisis de dominio institucional: la producción científica de la Universidad de Granada (SCI 1991-1999)". En: Revista española de documentación científica, 2005, v. 28, n. 2, pp. 170-195.

5. Grupo Scimago. Indicadores bibliométricos de la actividad científica española (1990-2004) [dirección Félix de Moya-Anegón, coordinación Zaida Chinchilla-Rodríguez, equipo de investigación Elena Corera-Álvarez [et al.] Madrid: Fecyt, s.a. Disponible:

http://sise.fecyt.es/Estudios/Doc/indicadores_bibliometricos.pdf [Consulta: 28/3/2007]

6. Van Raan, A. Advanced bibliometric methods for the evaluation of universities. En: Scientometrics, 1999 , v. 45 , n. 3, p. 417-423.

7. Grupo Scimago. "Producción ISI y tramos de investigación: cómo combinarlos con un nuevo indicador". En: El Profesional de la Información, 2006, v. 15, n. 3, p. 227-228.

8. INE (2006). Estadística de Enseñanza Universitaria, serie Profesorado de las universidades públicas/privadas y centros adscritos por universidades, sexo y situación laboral/dedicación. [En línea]. Madrid: Instituto Nacional de Estadística. Disponible: http://www.ine.es/inebase/ [Consulta: 20-04-2007].

Grupo Scimago (Imago scientiae o visualización de la ciencia)

scimago@ugr.es

http://www.scimago.es

http://www.atlasofscience.net

\section{¿Recibes ya EPI en casa?}


"Es fundamental que siga a los expertos en mi campo."

Con el Identificador de Autor de Scopus puedo acceder de manera rápida y sencilla a una visión global de los articulos escritos por los autores más importantes.

Ya no tengo que revisar el texto completo para confirmar su identidad.

Su universidad tiene acceso a Scopus. ¡Pruébelo! Le gustará.

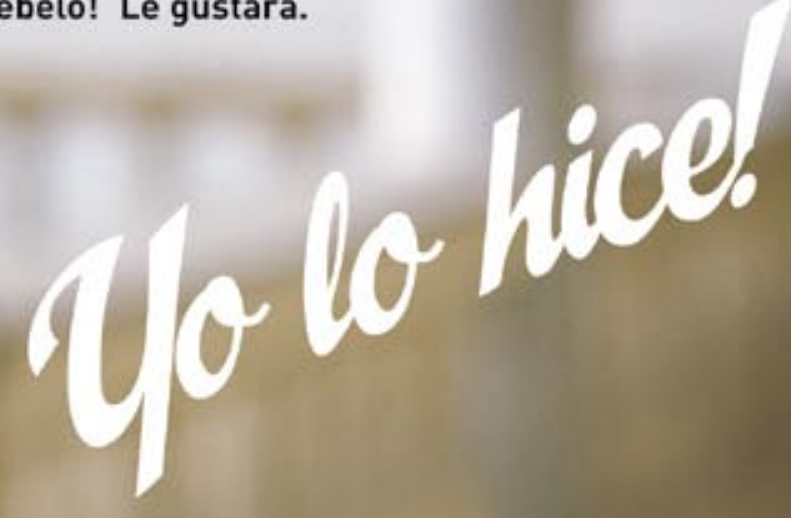

- Scopus es la mayor base de datos de resúmenes y citas de literatura cientifica y recursos de calidad de la web del mundo.

- Puede enlazar de manera sencilla a artículos a texto completo y a otros recursos de la biblioteca para obtener una visión general de las tendencias en su campo de investigación.

Benjamin Vargas-Quesada

Profesor Asociado - Library and Information Science

Universidad de Granada, España 